\title{
SURGERY ON RICCI POSITIVE MANIFOLDS
}

\author{
David Wraith \\ School of Mathematics, Trinity College, Dublin, Ireland. \\ wraith@maths.tcd.ie
}

\begin{abstract}
We consider performing surgery on Riemannian manifolds with positive Ricci curvature. We find conditions under which the resulting manifold also admits a positive Ricci curvature metric. These conditions involve dimension and the form taken by the metric in a neighbourhood of the surgery.

AMS subject classifications: Primary: 53C20 Secondary: 57R65

Keywords: positive Ricci curvature, surgery.
\end{abstract}

Submitted 21/8/97 


\section{§o. INTRODUCTION}

In this paper we prove a surgery result for manifolds of positive Ricci curvature. The relevance of surgery to questions of curvature was first seen in the following theorem, which concerns manifolds of positive scalar curvature (see [4] and [5]).

Theorem 0.1 (Gromov, Lawson, Schoen, Yau). Suppose $M$ is a manifold of dimension $\geq 5$ with a positive scalar curvature metric. Let $\hat{M}$ be the result of performing a surgery of codimension $\geq 3$ on $M$. Then $\hat{M}$ has a positive scalar curvature metric.

As well as generating new examples, this result has led to many structure theorems, including a complete classification of simply connected manifolds admitting positive scalar curvature metrics in dimensions $\geq 5$. It has been conjectured that a similar result might be true in the realm of positive Ricci curvature, perhaps involving an increased codimension condition. However, proving such a result would appear a very difficult proposal.

A limited Ricci positive surgery theorem was proved by Sha and Yang in [6]. We describe this presently.

Suppose we are given a Riemannian manifold $M^{n+m}$ having positive Ricci curvature and an isometric embedding $\iota: D_{R}^{m+1}(N) \times S^{n-1}(\rho) \longrightarrow M$ where $D_{R}^{m+1}(N)$ denotes a geodesic ball of radius $R$ in the $(m+1)$-sphere with the round metric of radius $N$. $S^{n-1}(\rho)$ is the $(n-1)$-sphere with the round metric of radius $\rho$.

We can regard $\iota$ as a trivialisation of the normal bundle of $\iota\left(\{0\} \times S^{n-1}\right)$.

Theorem 0.2 (Sha, Yang). Let $\hat{M}$ be the result of performing surgery on $\iota\left(\{0\} \times S^{n-1}\right)$ using the trivialisation $\iota$, and assume $n, m+1 \geq 3$. Then there exists $\kappa\left(n, m, R N^{-1}\right)>0$ such that if $\frac{\rho}{N}<\kappa$ then $\hat{M}$ can be equipped with a Ricci positive metric.

It is natural to ask if Sha and Yang's result remains true if the trivialisation $\iota$ is replaced by some other trivialisation. Of course, changing the normal bundle trivialisation will in general alter the homotopy type of the resulting manifold.

Suppose we have the following:

(i) A Riemannian manifold $\left(M^{n+m}, g\right)$ with positive Ricci curvature.

(ii) An isometric embedding $\iota: D_{R}^{m+1}(N) \times S^{n-1}(\rho) \longrightarrow M$ where $D_{R}^{m+1}(N)$ denotes a geodesic ball of radius $R$ in the $(m+1)$-sphere with the round metric of radius $N . S^{n-1}(\rho)$ is the $(n-1)$-sphere with the round metric of radius $\rho$. 
(iii) A smooth map $T: S^{n-1} \longrightarrow S O(m+1)$. This induces a map

$$
\begin{gathered}
\tilde{T}: D^{m+1} \times S^{n-1} \longrightarrow D^{m+1} \times S^{n-1} \\
(r, y, x) \mapsto(y, T(x) y, x)
\end{gathered}
$$

where we are thinking of $D^{m+1}$ in polar coordinates $(r, y): r \in[0, R], y \in S^{m}$.

Define the manifold $\hat{M}$ to be

$$
\hat{M}=\left(M \backslash \iota\left(D_{R}^{m+1}(N) \times S^{n-1}(\rho)\right) \amalg\left(D^{n} \times S^{m}\right) / \sim\right.
$$

where $\stackrel{\circ}{D}$ denotes the interior and $\sim$ indicates the identification of boundaries using $\tilde{T} \circ \iota$ in the obvious manner.

Observe that this construction is just a surgery on $\iota\left(\{0\} \times S^{n-1}\right)$ using the trivialisation $\tilde{T} \circ \iota$. Moreover, the effect of performing surgery on $\iota\left(\{0\} \times S^{n-1}\right)$ using any normal bundle trivialisation can be realised in this way, by a careful choice of $T$.

We can now state our main theorem.

Theorem 0.3. Suppose we have $M, \iota$ and $T$ as specified by $(i)-($ iii) above. Suppose further that $n \geq m+1 \geq 3$. There exists $\kappa\left(n, m, R N^{-1}, T\right)>0$ such that if $\frac{\rho}{N}<\kappa$ then we can choose a Riemannian metric on $D^{n} \times S^{m}$ with positive Ricci curvature which gels with the metric on

$$
M \backslash \iota\left(D_{R}^{m+1}(N) \times S^{n-1}(\rho)\right)
$$

to give a Ricci positive metric on $\hat{M}$.

The next proposition says that we can arrange for our metric on $D^{n} \times S^{m}$ to have further nice properties. Let $\pi: D^{n} \times S^{m} \longrightarrow \mathbb{R}$ represent projection onto the radial coordinate of $D^{n}$.

Proposition 0.4. Suppose we have $M, \iota$ and $T$ as before. Given $\kappa_{1}>0$ there exists a $\kappa\left(n, m, T, R N^{-1}, \kappa_{1}\right)>0$ such that if $\frac{\rho}{N}<\kappa$ we can choose the metric on $D^{n} \times S^{m}$ in Theorem 0.3 so that $\pi^{-1}\left[0, R_{1}\right]$ is isometric to $D_{R_{1}}^{n}\left(N_{1}\right) \times S^{m}\left(\rho_{1}\right)$ for some $R_{1}, \rho_{1}$ and $N_{1}$, with $\frac{\rho_{1}}{N_{1}}<\kappa_{1}$ and $\frac{R_{1}}{N_{1}}=\Delta$, a fixed constant independent of $M, \iota, T$ and $\kappa_{1}$.

The following result is a consequence of Theorem 0.3 :

Theorem 0.5. Let $M^{q}$ be a manifold admitting a positive Ricci curvature metric. Let $\bar{M}$ be the total space of a bundle with base $M$ and fibre $S^{k}, k \geq q-1 \geq 2$. If $\hat{M}$ is the result of performing surgery on a fibre sphere of $\bar{M}$, then $\hat{M}$ admits a positive Ricci curvature metric.

These results are of particular interest in the context of exotic spheres. This subject is explored in a separate paper [7].

The remainder of the paper is mainly dedicated to proving Theorem 0.3 . This amounts to constructing a metric on $D^{n} \times S^{m}$ with certain properties. In $\S 1$ we describe the type of 
metrics under consideration, and make explicit the properties they must satisfy. In $\S 2$ we perform the metric construction and in $\S 3$ we give the proofs of Theorem 0.3 , Proposition 0.4 and Theorem 0.5 .

The author would like to thank Stephan Stolz for suggesting this problem and for many valuable conversations.

\section{§1. GENERAL FORM OF THE METRIC}

The Riemannian metrics we construct in this paper are all submersion metrics. For a full exposition of the theory of these objects, see [1, Chapter 9]. Riemannian submersions are relevant to our discussion because their Ricci curvature is relatively straightforward to compute using the O'Neill formulae (see [1, Proposition 9.36])

We introduce a method of constructing submersion metrics which is due to Vilms $[1$, Theorem 9.59].

Construction 1.1. Let $G$ be a Lie group and $P \longrightarrow B$ a principal $G$-bundle. Let $F$ be a manifold with a smooth (left) $G$-action and let

$$
E=P \times_{G} F \longrightarrow B
$$

be the associated fibre bundle over $B$ with fibre $F$. Suppose we have:

i) a metric $\hat{g}$ on $F$ which is equivariant with respect to the $G$-action,

ii) a metric $\breve{g}$ on $B$,

iii) a connection on $P$

(iv) a smooth function $\mu: B \longrightarrow \mathbb{R}^{+}$

then there exists a unique submersion metric on $E$ with horizontal distribution is associated to the connection, and given $b \in B,\left(F_{b}, g_{b}\right)$ is (non-canonically) isometric to $\left(F, \mu^{2} \hat{g}\right)$.

The choice of principal connection clearly influences the Ricci curvature of the resulting submersion metric. The following lemma describes, in effect, a way of rendering this contribution insignificant. The proof is a direct consequence of the O'Neill formulae.

Lemma 1.2. Suppose we have a fibre bundle which has been equipped with a submersion metric via Construction 1.1. If the base is not compact then suppose further that the connection is flat outside some compact set. Let us scale the metric in fibre directions by $t \lambda^{2}$, where $\lambda: B \longrightarrow \mathbb{R}^{+}$and $t>0$. Let $r_{t \lambda^{2}}$ denote the Ricci tensor of this new metric. As $t$ approaches zero we have:

$$
\begin{gathered}
\lim _{t \rightarrow 0} r_{t \lambda^{2}}(X, U)=0 \\
\lim _{t \rightarrow 0} r_{t \lambda^{2}}(X, Y)=\check{r}(X, Y)-\frac{\operatorname{dim} F}{\lambda} \operatorname{Hess}_{\lambda}(X, Y) \\
\lim _{t \rightarrow 0} r_{t \lambda^{2}}(U, V)=\hat{r}_{b}(U, V)
\end{gathered}
$$

where $X$ and $Y$ are horizontal vectors, $U$ and $V$ are vertical vectors, $\check{r}$ denotes the Ricci tensor of the base, $\hat{r}_{b}$ the Ricci tensor on the (unscaled) fibre $F_{b}$ and $\operatorname{Hess}_{\lambda}(X, Y)$ the Hessian of $\lambda$ on the base. 
Corollary 1.3. In the metric construction of Lemma 1.2, we will produce a Ricci positive metric on the total space for some suitably small $t$ provided the Ricci curvature of the fibre $F$ is strictly positive and that of the warped product $B \times_{\lambda^{2}} F$ is strictly positive in $B$ directions.

\section{Proof.}

The right hand side of the second limit formula coincides precisely with the curvature in base directions of the warped product $B \times_{\lambda^{2}} F$ (as given by the O'Neill formulae).

Note that without the compactness assumption, we cannot necessarily find a $t$ to give a Ricci positive metric on the entire total space.

\section{Submersion Metrics on $D^{n} \times S^{m}$}

With Construction 1.1 in mind, let us address the problem of assigning metrics to $D^{n} \times S^{m}$. We will regard this product as a bundle with base $D^{n}$ and fibre $S^{m}$. First we show how to choose the fibre and base metrics, then go on to choose a principal connection.

\section{The Fibre Metrics}

We will assume that each fibre is equipped with the round metric of radius 1 , but allow warping by a positive real valued function $f$ defined on the base.

\section{The Base Metric}

It will be convenient to describe the base, $D^{n}$, in polar coordinates. In this setting the metric on a geodesic disk in the $n$-sphere of radius $N$ takes the form $d r^{2}+N^{2} \sin ^{2}\left(\frac{r}{N}\right) d s_{n-1}^{2}$.

We will denote by $a$ the radius of $D^{n}$, measured with respect to the metric $d r^{2}$. We remark now that $a>2$; a suitable value will be determined later.

The metric we choose for the base takes the form $d r^{2}+h^{2}(r) d s_{n-1}^{2}$ for some function

$h:[0, a] \longrightarrow \mathbb{R}^{+}$. Initially, though, we will regard $h$ as a map $\mathbb{R}^{+} \longrightarrow \mathbb{R}^{+}$. After finding a value for $a$, we then restrict to the above interval.

\section{The Connection}

In order to ensure smooth metric gluing in the surgery process, our connection must depend on the 'twist' map $T$. We choose as follows.

Let $A^{n}$ denote the annular region $[2, a] \times S^{n-1} \subset D^{n}$. Note that by rotational symmetry, radial distance on $D^{n}$ is well-defined independent of our particular choice of base metric.

Consider the following maps:

$$
\begin{gathered}
\mathcal{T}: A^{n} \times S^{m} \longrightarrow A^{n} \times S^{m} \\
(r, x, y) \mapsto(r, x, T(x) y)
\end{gathered}
$$

and the corresponding map of associated principal $S O(m+1)$ bundles: 


$$
\begin{gathered}
\mathcal{T}_{1}: A^{n} \times S O(m+1) \longrightarrow A^{n} \times S O(m+1) \\
(r, x, z) \mapsto(r, x, T(x) . z)
\end{gathered}
$$

where the dot denotes group multiplication.

Pull back the trivial principal $S O(m+1)$-connection on $A^{n} \times S O(m+1)$ using $\mathcal{T}_{1}$ to obtain a new connection $\nabla_{1}$ on $A^{n} \times S O(m+1) . \nabla_{1}$ is flat as it is the pull-back of a flat connection.

By including $A^{n} \times S O(m+1)$ in $D^{n} \times S O(m+1)$ we obtain a principle connection $\nabla$ on $D^{n} \times S O(m+1)$ by extending $\nabla_{1}$. We do this in such a way that $\nabla$ is flat at radii $r \in[0,1]$. Note that in general, it is not possible to achieve a flat extension to all radii.

The crucial point about this construction is that the map $\tilde{T}$ restricted to a neighbourhood of the boundary of $D_{N}^{m+1}(R) \times S^{n-1}(\rho)$ coincides with the map $\mathcal{T}$ restricted to a neighbourhood of the outer boundary of $A^{n} \times S^{m}$. It is this fact that enables smooth metric surgery to be performed, provided the scaling functions $f$ and $h$ are properly chosen.

Construction 1.1 now gives us a metric on $D^{n} \times S^{m}$. Because of our choice of connection, this metric will be a warped product of the form $d r^{2}+h^{2}(r) d s_{n-1}^{2}+f^{2}(r) d s_{m}^{2}$ for $r \in[0,1]$ and $r \in[2, a]$. Let us at this point state the formulae for the Ricci curvature of such a warped product.

The tangent space at any point splits into three mutually othogonal subspaces corresponding to the radial direction, the $S^{n-1}$ direction and the $S^{m}$ direction. From now on, $X$ and $Y$ and $U$ will denote unit vectors in each of these three subspaces respectively.

The Ricci curvatures are as follows:

$$
\begin{gathered}
r(X)=-(n-1) \frac{h^{\prime \prime}}{h}-m \frac{f^{\prime \prime}}{f} \\
r(Y)=-\frac{h^{\prime \prime}}{h}+(n-2) \frac{1-\left(h^{\prime}\right)^{2}}{h^{2}}-m \frac{h^{\prime} f^{\prime}}{h f} \\
r(U)=-\frac{f^{\prime \prime}}{f}+(m-1) \frac{1-\left(f^{\prime}\right)^{2}}{f^{2}}-(n-1) \frac{h^{\prime} f^{\prime}}{h f}
\end{gathered}
$$

In terms of the associated bilinear form we also have

$$
r(X, Y)=r(Y, U)=r(U, X)=0
$$

Observation 1.4. If the warped product metric $d r^{2}+h^{2} d s_{n-1}^{2}+f^{2} d s_{m}^{2}$ has positive Ricci curvature, then shrinking fibres (ie multiplying the function $f$ by a small constant) has no effect on the Ricci curvature in base directions, and increases the Ricci curvature in fibre directions. (Compare Lemma 1.2). 


\section{Restrictions on the Metric}

With Proposition 0.4 in mind, we impose some conditions on the functions $h$ and $f$ near $r=0$ :

\section{Condition $A$}

There exists $R^{\prime} \in(0,1)$ such that for all $r<R^{\prime}, f(r)$ and $h(r)$ satisfy

(1) $f(r)>0$

(2) $f^{\prime}(r)=0$

(3) $h(r)=N^{\prime} \sin \frac{r}{N^{\prime}} \quad\left(\right.$ some $\left.N^{\prime}>0\right)$

We will denote this constant value of $f$ by $\rho^{\prime}$.

Since our metric must gel smoothly with the metric on $M \backslash \iota\left(D_{R}^{m+1}(N) \times S^{n-1}(\rho)\right)$ this means $h$ and $f$ should satisfy some conditions near the boundary of $D^{n} \times S^{m}$. We will define $f$ and $h$ as functions on the whole of $\mathbb{R}^{+}$and impose the following condition:

\section{Condition $B$}

There exists $a>2$ such that for $r \in(a-\epsilon, a]$

(1) $f(r)=N \sin \left(\frac{R-a+r}{N}\right)$

(2) $h(r) \equiv \rho$

To understand $B(1)$, notice that the points of distance $R$ from a given point in a round $n$-sphere of radius $N$ form a round $(n-1)$-sphere of radius $N \sin \frac{R}{N}$.

\section{Condition $C$}

The metric on $D^{n} \times S^{m}$ should be Ricci positive.

At this point recall the result of Ehrlich [3] which states that a metric on a complete manifold with non-negative Ricci curvature which is strictly positive in some region can be deformed to a metric with strictly positive Ricci curvature everywhere. As a consequence of this we can replace condition $C$ by

\section{Condition $C^{\prime}$}

The metric on $D^{n} \times S^{m}$ should be Ricci non-negative and strictly positive in some region.

In practice, it turns out to be easier to replace condition $B$ by the following alternative condition: 


\section{Condition $B^{\prime}$}

There exists $b>2$ such that for all $r>b$

(1) $1>f^{\prime}(r)>\cos \frac{R}{N}$

(2) $h^{\prime}(r) \equiv 0$

(3) $f^{\prime \prime}(r) \equiv 0$

We must explain the relevance of this to condition $B$. First, though, we have:

Observation 1.5. Suppose we have two concave down functions:

$$
\begin{aligned}
& \theta_{1} \text { defined for } r \leq r_{1} \\
& \theta_{2} \text { defined for } r \geq r_{2}
\end{aligned}
$$

If $r_{1}<r_{2}$ and both $\theta_{1}$ and $\theta_{2}$ have positive slope, then we can make a concave down extension over the interval $\left[r_{1}, r_{2}\right]$ to give a single smooth function provided:

$$
\theta_{1}\left(r_{1}\right)<D \theta_{2}\left(r_{2}\right)\left(r_{1}\right)
$$

and

$$
\theta_{2}\left(r_{2}\right)<D \theta_{1}\left(r_{1}\right)\left(r_{2}\right)
$$

where $D \theta_{i}(r): \mathbb{R} \longrightarrow \mathbb{R}$ is the linear approximation to $\theta_{i}$ at the point $r$ (ie the function whose graph is the tangent line to $\theta_{i}$ at $r$ ).

We now show, by making deformations to $f$, how condition $B$ can be achieved assuming condition $B^{\prime}$ without introducing negative curvature.

Assume $B^{\prime}(2)$ holds. If we insist on the Ricci curvature being positive, it is clear from the formulae above that this is the same as demanding $f^{\prime \prime}<0<f^{\prime}<1$. The last of these inequalities is the left hand side of $B^{\prime}(1)$. With this in mind, consider a first quadrant function $f$, satisfying both $f^{\prime \prime}=0\left[B^{\prime}(3)\right]$ and $f^{\prime}>0$ for $r>b$. We address the problem of deforming $f$ so as to join smoothly with the function $N \sin \left(\frac{R-a+r}{N}\right)$ at the point $r=a$ $(>b)$.

Lemma 1.6. The two conditions of Observation 1.5 can be satisfied for $f$ at $r \leq b$ and $N \sin \left(\frac{R-a+r}{N}\right)$ at $r \geq a$, some $a>b$ if:

$$
f^{\prime}(b)>\cos \frac{R}{N}
$$

and

$$
f(b)<N \sin \frac{R}{N}
$$

Note that the first of these conditions justifies the right hand inequality of $B^{\prime}(1)$. 


\section{Proof.}

At $r=a, N \sin \left(\frac{R-a+r}{N}\right)$ has the value $N \sin \frac{R}{N}$, and the tangent line has slope $\cos \frac{R}{N}$. As a result of $(* *)$ and the fact that $\cos \frac{R}{N}>0$, there is a point $x>b$ such that the linearisation

$$
D\left[N \sin \left(\frac{R-x+r}{N}\right)\right](x)(b)=f(b)
$$

Condition (ii) of Observation 1.5 is clearly satisfied if we put $r_{2}=x$, as $f^{\prime}(b)>\cos \frac{R}{N}$ by (*). Now at any point $r_{2}<x$, condition $(i)$ holds. By the openness of condition (ii), we are then able to choose a point $a<x$ where condition (ii) is still satisfied.

We have seen, then, that condition $B^{\prime}$ is almost sufficient to be able to guarantee satisfying condition $B$ without introducing negative curvature. We still need to ensure, however, that $f\left(a^{\prime}\right)<N \sin \frac{R}{N}$ and $h(r)=\rho$ for $r \geq a^{\prime}[B(2)]$.

Definition 1.7. Let $\kappa=\frac{h(b)}{f(b)} \sin \frac{R}{N}$.

Lemma 1.8. If $\frac{\rho}{N}<\kappa$ then we can ensure $f(b)<N \sin \frac{R}{N}$ and $h(r)=\rho$ for $r \geq b$.

\section{Proof.}

Thinking in terms of the graphs of $f$ and $h$, we can achieve $h(b)=\rho$ by simply reparameterising both axes by a factor of $\frac{\rho}{h(b)}$. (This amounts to performing a global rescale, which has no effect on either the Ricci curvature or the ratios $\frac{R}{N}$ and $\frac{R^{\prime}}{N^{\prime}}$.) We then only need to ensure the following inequality is satisfied:

$$
f(b) \cdot \frac{\rho}{h(b)}<N \sin \frac{R}{N}
$$

and we can rearrange this to give

$$
\frac{\rho}{N}<\kappa
$$

Corollary 1.9. If $\frac{\rho}{N}<\kappa$ then we can deform functions $h$ and $f$ satisfying condition $B^{\prime}$ to satisfy condition $B$ without introducing negative Ricci curvature.

\section{$\S 2$ The Construction}

In the light of Lemma 1.2 it suffices to focus attention on warped product metrics $d r^{2}+h^{2}(r) d s_{n-1}^{2}+f^{2}(r) d s_{m}^{2}$ on $\mathbb{R}^{+} \times S^{n-1} \times S^{m}$, on the understanding that $f$ is sufficiently 'squashed' at least over the interval $[1,2]$ to ensure that the influence of the connection does not upset the Ricci positivity when we use the Vilms construction. Of course, the functions $f$ and $h$ must satisfy all the boundary conditions specified in the last section. 
We will construct $f$ and $h$ via a sequence of approximations. We break the process into four steps.

\section{STEP 1}

Definition 2.1. The function $f_{0}$ is defined by the $O D E$

$$
\begin{aligned}
& f_{0}^{\prime \prime}=f_{0}^{-1} \\
& f_{0}^{\prime}(0)=0 \\
& f_{0}(0)=1
\end{aligned}
$$

Observe that $f_{0}^{\prime}(r)=\sqrt{2 \ln f_{0}(r)}$ and so $\lim _{r \rightarrow \infty} f_{0}^{\prime}(r)=\infty$.

Definition 2.2. The function $h_{0}$ is defined by

$$
h_{0}(r)=f_{0}^{\prime}(r)
$$

A simple calculation using (1.1) and (1.2) yields the following:

Lemma 2.3. The metric $d r^{2}+h_{0}^{2}(r) d s_{n-1}^{2}+f_{0}^{2}(r) d s_{m}^{2}$ on $\mathbb{R}^{+} \times S^{n-1} \times S^{m}$ has strictly positive Ricci curvature in $\mathbb{R}^{+}$and $S^{n-1}$ directions.

In future we will refer to these directions as 'base-radial' and 'base-spherical' respectively. We postpone a discussion of fibre direction curvature until Step 3.

\section{STEP 2}

In this step we deform $f_{0}$ and $h_{0}$ to functions $f_{1}$ and $h_{1}$ which satisfy condition $A$. We deal first with $f_{0}$.

Choose a function $\tilde{f}_{0}(r)$ which satisfies the following:

1) $\tilde{f}_{0}(r)>0$

2) $\tilde{f}_{0}^{\prime}(r)=0$ for $r \in\left[0, \frac{1}{4}\right]$

3) $\tilde{f}_{0}^{\prime \prime}(r) \geq 0$

4) $\tilde{f}_{0}(r)=f_{0}(r)$ for $r \geq \frac{1}{2}$

Definition 2.3. Let $f_{1}(r)=\tilde{f}_{0}(r)+\alpha$ for some constant $\alpha>0$.

We will determine a suitable value for $\alpha$ in the following lemma. 
Lemma 2.4. There exists $\alpha>0$ so that the warped product metric $d r^{2}+h_{0}^{2}(r) d s_{n-1}^{2}+$ $f_{1}^{2}(r) d s_{m}^{2}$ has strictly positive Ricci curvature in base directions.

\section{Proof.}

First of all notice that the Ricci curvature of the metric increases in base-spherical directions when we replace $f_{0}$ by $f_{1}$. We therefore focus our attention on base-radial directions.

We want the inequality $-(n-1) \frac{h_{0}^{\prime \prime}}{h_{0}}-m \frac{f_{1}^{\prime \prime}}{f_{1}} \geq 0$ to be satisfied, so by the ansatz this means we need to keep

$$
f_{0}^{-2} \geq \frac{m}{n-1} \frac{\tilde{f}_{0}^{\prime \prime}}{\tilde{f}_{0}+\alpha}
$$

Since $n-1 \geq m$ and $\frac{\tilde{f}_{0}^{\prime \prime}}{\tilde{f}_{0}}=f_{0}^{-2}$ for $r \geq \frac{1}{2}$ the inequality is true for these values of $r$ and for all $\alpha>0$. By compactness, we can then choose a suitably large value for $\alpha$ so that the inequality remains true over $\left[0, \frac{1}{2}\right]$.

Choose a number $N^{\prime}>0$ (any will do). The following Lemma is an easy consequence of Observation 1.5 .

Lemma 2.5. There exists a concave down function $h_{1}$ with the following properties:

1) $h_{1}(r)=N^{\prime} \sin \frac{r}{N^{\prime}}$ for $r \in\left[0, R^{\prime}\right]$, some $R^{\prime}<\frac{1}{4}$

2) $h_{1}(r)=h_{0}(r)$ for $r \geq \frac{1}{2}$

Since $f_{1}$ is constant over $\left[0, \frac{1}{4}\right]$ it is clear from (1.1) - (1.3) that the metric $d r^{2}+$ $h_{1}^{2} d s_{n-1}^{2}+f_{1}^{2} d s_{m}^{2}$ has strictly positive Ricci curvature if and only if $h_{1}^{\prime \prime}<0<h_{1}^{\prime}<1$.

\section{STEP 3}

We now investigate the Ricci curvature in fibre directions. In particular we study the effects of shrinking $f_{1}$ by a constant factor.

Lemma 2.6. Given any $l>0$ we can choose $k$ such that $d r^{2}+h_{1}^{2} d s_{n 01}^{2}+k^{2} f_{1}^{2} d s_{m}^{2}$ has positive Ricci curvature at all points with radial coordinate $\leq l$.

Definition 2.7. Let $R i c_{k}$ denote the Ricci curvature of the metric $d r^{2}+h_{1}^{2} d s_{n-1}^{2}+$ $k^{2} f_{1}^{2} d s_{m}^{2}$.

\section{Proof of Lemma 2.6.}

First of all notice that by (1.1) and (1.2) $k$ has no effect on base-direction curvatures. We therefore focus on the Ricci curvature in fibre directions.

By Observation 1.4 we can guarantee positivity in fibre directions at radii $\leq 1$ by choosing $k$ sufficiently small. 
Consider radii $>1$. For these values of $r$ we have

$$
\begin{aligned}
& f_{1}=f_{0}+\alpha \\
& h_{1}=h_{0}
\end{aligned}
$$

Note that by integrating $f_{0}^{\prime} f_{0}^{\prime \prime}$ by parts and using the ansatz we have

$$
f_{0}^{\prime}=\sqrt{2 \ln f_{0}}
$$

Using these in (1.3) we obtain

$$
\operatorname{Ric}_{k}(U)=\frac{m-1}{\left(f_{0}+\alpha\right)^{2}}\left[\frac{1}{k^{2}}-2 \ln f_{0}\right]-\frac{n}{f_{0}\left(f_{0}+\alpha\right)}
$$

and the assertion of the Lemma follows.

Since $\lim _{r \rightarrow \infty} f_{0}(r)=\infty$ we see that $\operatorname{Ric}_{k}(U)$ will be strictly negative for suitably large $r$. This will certainly be the case, for example, when $f_{0}(r)>e^{\frac{1}{2 k^{2}}}$.

Definition 2.8. Let $z_{k}$ be the smallest value of $r$ for which $\operatorname{Ric}_{k}(U)=0$.

\section{Lemma 2.9.}

(i) $z_{k} \longrightarrow \infty$ as $k \longrightarrow 0$.

(ii) $k f_{1}^{\prime}\left(z_{k}\right) \longrightarrow 1$ as $k \longrightarrow 0$.

\section{Proof.}

(i) is obtained from the expression for $\operatorname{Ric}_{k}(U)$.

To see (ii): assuming $z_{k}>1$ we have

$$
k f_{1}^{\prime}\left(z_{k}\right)=k f_{0}^{\prime}\left(z_{k}\right)=\sqrt{1-\frac{k^{2} n}{m-1} \cdot \frac{f_{0}\left(z_{k}\right)+\alpha}{f_{0}\left(z_{k}\right)}}
$$

Now use (i).

Fix a value $K$ such that $K f_{1}^{\prime}\left(z_{K}\right)>\cos \frac{R}{N}$.

Definition 2.10. Let $f_{2}(r)=K f_{1}(r)$.

Let us summerise our gains at this point:

Corollary 2.11. The metric $d r^{2}+h_{1}^{2} d s_{n-1}^{2}+f_{2}^{2} d s_{m}^{2}$ satisfies condition $A$, condition $C$ for $r \in\left[0, z_{K}\right)$ and condition $B^{\prime}(1)$ for $r \in\left(z_{K}-\epsilon, z_{K}\right)$, some $\epsilon>0$.

\section{STEP 4}

In this step we explain how to deform $f_{2}$ and $h_{1}$ to satisfy the remaining boundary conditions $B^{\prime}(2)$ and $B^{\prime}(3)$ without introducing negative Ricci curvature. The resulting functions are then the desired functions $f$ and $h$.

To achieve $B^{\prime}(2)$ and $B^{\prime}(3)$ we have to 'straighten' $f_{2}$ and $h_{1}$. First we deal with $f_{2}$.

Choose a point $p<z_{K}$ for which $f_{2}^{\prime}(p)>\cos \frac{R}{N}$. The following Lemma is obvious: 
Lemma 2.12. Given $\epsilon>0$ we can smoothly deform $f_{2}$ over $[p, p+2 \epsilon]$ to give a function $f_{2, \epsilon}$ with the following properties:

1) $f_{2, \epsilon}^{\prime \prime}(r)=0$ for $r \in[p+\epsilon, p+2 \epsilon]$

2) $f_{2, \epsilon}^{\prime \prime \prime}(r) \leq 0$ for $r \in[p, p+2 \epsilon]$

3) $f_{2}(p)-\epsilon \leq f_{2, \epsilon}(r) \leq f_{2}(p)+\epsilon$ for $r \in[p, p+\epsilon]$

4) $f_{2}^{\prime}(p)-\epsilon \leq f_{2, \epsilon}^{\prime}(r) \leq f_{2}^{\prime}(p)+\epsilon$ for $r \in[p, p+2 \epsilon]$

We define $f_{2, \epsilon}(r)$ for values of $r>p+2 \epsilon$ by linear extension.

Corollary 2.13. There exists $\epsilon>0$ such that the metric $d r^{2}+h_{1}^{2} d s_{n-1}^{2}+f_{2, \epsilon}^{2} d s_{m}^{2}$ has strictly positive Ricci curvature at all radii $r \in[0, p+2 \epsilon]$.

\section{Proof.}

By choosing $\epsilon$ suitably small we can ensure $p+2 \epsilon<z_{K}$. Moreover, by Lemma 2.12 we can bound

$$
\max \left(\frac{f_{2, \epsilon}^{\prime \prime}(r)}{f_{2, \epsilon}(r)}-\frac{f_{2}^{\prime \prime}(r)}{f_{2}(r)}\right) \quad \text { and } \quad\left|\frac{f_{2, \epsilon}^{\prime}(r)}{f_{2, \epsilon}(r)}-\frac{f_{2}^{\prime}(r)}{f_{2}(r)}\right|
$$

in terms of $\epsilon$ for $r \in[p, p+2 \epsilon]$. Therefore, choosing $\epsilon$ smaller if necessary, it is clear from (1.1) - (1.3) that we can achieve Ricci positivity over the given interval.

Choose and fix a number $\epsilon_{1}$ such that the conclusion of Corollary 2.13 is true for $\epsilon=\epsilon_{1}$.

Definition 2.14. Let $f(r)=f_{2, \epsilon_{1}}(r)$

We now turn our attention to $h_{1}$. We perform our deformation of $h_{1}$ over the interval $\left[p+\epsilon_{1}, p+2 \epsilon_{1}\right]$ (and then extend linearly for higher values of $r$ ).

Lemma 2.15. Given $\epsilon^{\prime}$ with $0<\epsilon^{\prime}<\epsilon_{1}$ we can smoothly deform $h_{1}$ to a function $h_{1, \epsilon^{\prime}}$ over the interval $\left[p+\epsilon_{1}, p+2 \epsilon_{1}\right]$ which satisfies the following conditions:

1) $h_{1, \epsilon^{\prime}}^{\prime \prime}(r)=0$ for $r \in\left[p+\epsilon_{1}+\epsilon^{\prime}, p+2 \epsilon_{1}\right]$

2) $h_{1, \epsilon^{\prime}}^{\prime \prime \prime}(r) \leq 0$ for $r \in\left[p+\epsilon_{1}, p+2 \epsilon_{1}\right]$

3) $h_{1}\left(p+\epsilon_{1}\right)-\epsilon^{\prime} \leq h_{1, \epsilon^{\prime}}(r) \leq h_{1}\left(p+\epsilon_{1}\right)+\epsilon^{\prime}$ for $r \in\left[p+\epsilon_{1}, p+\epsilon_{1}+\epsilon^{\prime}\right]$

4) $h_{1}^{\prime}\left(p+\epsilon_{1}\right)-\epsilon^{\prime} \leq h_{1, \epsilon^{\prime}}^{\prime}(r) \leq h_{1}^{\prime}\left(p+\epsilon_{1}\right)+\epsilon^{\prime}$ for $r \in\left[p+\epsilon_{1}, p+\epsilon_{1}+\epsilon^{\prime}\right]$

Corollary 2.16. There exists $\epsilon^{\prime}<\epsilon_{1}$ such that the metric $d r^{2}+h_{1, \epsilon^{\prime}}^{2} d s_{n-1}^{2}+f^{2} d s_{m}^{2}$ has non-negative Ricci curvature for $r \in\left[p+\epsilon_{1}, p+2 \epsilon_{1}\right]$ ( and therefore for all $r \in \mathbb{R}^{+}$). 


\section{Proof.}

By Lemma 2.15 we can bound the quantity

$$
\left(\frac{h_{1, \epsilon^{\prime}}^{\prime}(r)}{h_{1, \epsilon^{\prime}}(r)}-\frac{h_{1}^{\prime}(r)}{h_{1}(r)}\right)
$$

in terms of $\epsilon^{\prime}$. From (1.1) - (1.3) we see that a suitably small choice of $\epsilon^{\prime}$ will therefore ensure the strict positivity of the Ricci curvature in base-spherical and fibre directions. In base-radial directions the Ricci curvature is $\geq 0$ because $f^{\prime \prime}(r)=0$ for $r \in\left[p+\epsilon_{1}, p+2 \epsilon_{1}\right]$ and $h_{1, \epsilon^{\prime}}^{\prime \prime}(r) \leq 0$.

Definition 2.17. Let $h(r)=h_{1, \epsilon^{\prime}}(r)$ for any value of $\epsilon^{\prime}$ for which the conclusion of Corollary 2.16 is true.

To complete this step we need to set the values of $a$ and $b$ (see conditions $B$ and $B^{\prime}$ respectively).

Definitions 2.18. Let $b$ be any (fixed) number greater than $p+2 \epsilon_{1}$, and let $a>b$ be any value at which condition $B$ can then be achieved.

We have therefore shown the following:

Corollary 2.19. The metric $d r^{2}+h^{2} d s_{n-1}^{2}+f^{2} d s_{m}^{2}$ satisfies conditions $A, B$ and $C^{\prime}$ for all $r \in[0, a]$.

\section{$\S 3$ Proofs of the main results.}

\section{Proof of Theorem 0.3.}

By Lemma 2.10 it is clear that the constant $k$ in Step 3 can be chosen arbitrarily small. By Lemma 1.2 we can therefore take the constant $K$ in Definition 2.11 to be so small that the metric defined by $f, h$ and the chosen connection using Construction 1.1 has strictly positive Ricci curvature at radii $\in[1,2]$. Corollary 1.9 together with Corollary 2.19 then shows the existence of a metric on $D^{n} \times S^{m}$ satisfying all the required properties.

\section{Proof of Proposition 0.4.}

It is clear from condition $A$ that we have an isometry $\pi^{-1}\left[0, R_{1}\right] \cong D_{N_{1}}^{n}\left(R_{1}\right) \times S^{m}\left(\rho_{1}\right)$ for some $R_{1}, \rho_{1}$ and $N_{1}$. Moreover, $R_{1}, \rho_{1}$ and $N_{1}$ differ from the quantities $R^{\prime}, \rho^{\prime}$ and $N^{\prime}$ in condition $A$ by a constant factor which arises from the 'gluing-in' process (see the proof of Lemma 1.8). In particular we have $\frac{R_{1}}{N_{1}}=\frac{R^{\prime}}{N^{\prime}}$ and $\frac{\rho_{1}}{N_{1}}=\frac{\rho^{\prime}}{N^{\prime}}$. 
Our choice of $N^{\prime}$ in Step 2 of the metric construction is arbitrary. Having made our selection, we are then free to choose any value for $R^{\prime}$ within an upper bound which depends only on $N^{\prime}$. In this way we can fix the quantity $\frac{R^{\prime}}{N^{\prime}}$ for every surgery we perform.

Recall that $\rho^{\prime}=f(0)=K f_{1}(0)$. We therefore have $\rho^{\prime} \longrightarrow 0$ as $K \longrightarrow 0$. Since we can assume $N^{\prime}$ is fixed for all surgeries, it is clear that by choosing $K$ sufficiently small we can construct a metric on $D^{n} \times S^{m}$ satisfying $\frac{\rho_{1}}{N_{1}}=\frac{\rho^{\prime}}{N^{\prime}}<\kappa_{1}$, for any $\kappa_{1}>0$.

\section{Proof of Theorem 0.5.}

By a result of [3] we can choose a Ricci positive metric on $M$ such that in some neighbourhood $U \subset M$ we have sectional curvature $K \equiv 1$. We now use Construction 1.1 to give us a metric on $\bar{M}$ : equip $S^{k}$ with the round metric of radius 1 , define $\mu: M \longrightarrow \mathbb{R}^{+}$ to be a constant function with value $c>0$, and choose a connection on the principle

bundle associated to $\bar{M}$ which is flat over $U$. It follows from Lemma 1.2 that by choosing $c$ sufficiently small we obtain a Ricci positive metric on $\bar{M}$ which is a product over $U$. Clearly, there is an isometric embedding $\iota: D_{N}^{n}(R) \times S^{m}(\rho) \longrightarrow \bar{M}$ with $N=1, \rho=c$ and some value of $R$ which is independent of $c$. Moreover, given any $\kappa>0$, by choosing $c$ smaller if necessary, we can always guarantee $\frac{\rho}{N}<\kappa$. The result now follows from Theorem 0.3 .

\section{REFERENCES}

[1] A. Besse, Einstein Manifolds, Springer-Verlag (1987).

[2] P. Ehrlich, Metric Deformations of Curvature, Geometriae Dedicata, 5, 1-23.

[3] L-Z Gau and S-T Yau, The Existence of Negatively Ricci Curved Metrics on Three Manifolds, Invent. Math. 85 (1986) 637-652.

[4] M. Gromov and H. B. Lawson, The Classification of Manifolds of Positive Scalar Curvature, Ann. of Math. 111 (1980), 423-434.

[5] R. Schoen and S-T Yau, On the Structure of Manifolds with Positive Scalar Curvature, Manuscripta Math. 28 (1979), 159-183.

[6] J-P Sha and D-G Yang, Positive Ricci Curvature on the Connected Sums of $S^{n} \times S^{m}$, J. Diff. Geom. 33 (1990), 127-138.

[7] D. Wraith, Exotic Spheres with Positive Ricci Curvature, J. Diff. Geom. 45 (1997). 